ARTICLE

DOI: $10.1038 / \mathrm{s} 41467-017-00175-6$

\title{
Programmable base editing of zebrafish genome using a modified CRISPR-Cas9 system
}

Yihan Zhang ${ }^{1,2}$, Wei Qin ${ }^{1}$, Xiaochan Lu¹, Jason Xu², Haigen Huang ${ }^{2}$, Haipeng Bai ${ }^{1}$, Song $\mathrm{Li}^{1}$ \& Shuo Lin ${ }^{1,2}$

Precise genetic modifications in model animals are essential for biomedical research. Here, we report a programmable "base editing" system to induce precise base conversion with high efficiency in zebrafish. Using cytidine deaminase fused to Cas 9 nickase, up to $28 \%$ of site-specific single-base mutations are achieved in multiple gene loci. In addition, an engineered Cas9-VQR variant with $5^{\prime}$-NGA PAM specificities is used to induce base conversion in zebrafish. This shows that Cas9 variants can be used to expand the utility of this technology. Collectively, the targeted base editing system represents a strategy for precise and effective genome editing in zebrafish.

\footnotetext{
${ }^{1}$ Laboratory of Chemical Genomics, School of Chemical Biology and Biotechnology, Peking University Shenzhen Graduate School, Shenzhen, 518055, China. ${ }^{2}$ Department of Molecular, Cell and Developmental Biology, University of California, Los Angeles, 90095 CA, USA. Yihan Zhang and Wei Qin contributed equally to this work. Correspondence and requests for materials should be addressed to S.L. (email: shuolin@ucla.edu)
} 
T he clustered regularly interspaced short palindromic repeat (CRISPR) system has been developed as a versatile genome editing tool in various organisms, including zebrafish ${ }^{1,2}$. The Cas9 protein, guided by guide RNA (gRNA), binds to the target DNA site on the genome and works as a nuclease to induce double-strand breaks (DSBs) ${ }^{1}$. As a natural cellular response, the DSBs are mainly repaired through the non-homologous end joining pathway. This process can generate random deletions or insertions. In addition, specific modifications, such as the substitutions of single bases, and the insertion of longer sequences like loxP elements, can be introduced into the genome with the presence of the homologous donor template through homologydirected repair $(\mathrm{HDR})^{3-5}$. Genome-wide association study coupled with the next-generation sequencing has identified a growing number of candidate genes with single-base mutations associated with human diseases. Inevitably, efficient methods are required to validate the causal mutations responsible for disease phenotypes $^{6}$. The most desirable approach is to introduce the human genetic mutations in model organisms by knock-in using the CRISPR-mediated HDR. Unfortunately, the efficiency of this donor-dependent HDR is low, which restricts the utility of this method $^{7}$.

Recently, a technology called "base editing" (BE) was reported, which enables direct and irreversible conversion of one targeted base to another in cultured mammalian cells in a programmable manner without the need for a $\mathrm{DSB}^{8}$. In this system, a cytidine deaminase was fused to the $\mathrm{N}$ terminus of a Cas9 nickase (nCas9), which mediates the direct conversion of $\mathrm{C} \rightarrow \mathrm{T}($ or $\mathrm{G} \rightarrow \mathrm{A}$ ) in human cells. The optimal deamination sites for this system are located in a $5 \mathrm{bp}$ window on the CRIPSR-Cas 9 target site, -17 to -13 upstream of the PAM sequence. Cas9 nickase maintains its activity to bind DNA with a gRNA and can only cut the non-edited strand, preventing DSBs. By nicking the non-edited DNA strand, both the newly synthesized DNA and damaged DNA are stimulated to resolve the U:G mismatch into T:A, improving the base conversion efficiency. In order to prevent $\mathrm{U}: \mathrm{G}$ to C:G reversion, a UDG inhibitor (UGI) from Bacillus subtilis bacteriophage PBS1 was fused to the C terminus of nCas9. With this design, it is reported that the $\mathrm{BE}$ system can achieve permanent correction of $15-75 \%$ of total cellular DNA with minimal (typically $\leq 1 \%$ ) indel formation ${ }^{8}$. Conceptually, the $\mathrm{BE}$ system should have great potential applications in gene editing by introducing single-base changes to correct or mimic mutations of human genetic disorders in model animals. To date, this system has been reported to work in mouse and several crops $^{9-11}$. However, it has not been tested if this system will work in zebrafish.

Here, we demonstrate that this $\mathrm{BE}$ system can achieve base substitution at efficiency between 9.25 and $28.57 \%$ with very low indel formation in zebrafish. To enrich the toolbox of this $\mathrm{BE}$ system, we also replace the Cas9 nickase with VQR variant nickase, which recognizes the $5^{\prime}$-NGA PAM. Sequencing results indicate that this $\mathrm{BE}-\mathrm{VQR}$ system also induce efficient base substitution in a targeted manner. Overall, we demonstrate that the deaminase-Cas9 tool of "base editing" provides a simple and efficient method for introducing single-base changes in zebrafish.

\section{Results}

BE system can induce base conversion in zebrafish. To explore whether the BE (rAPOBEC1-XTEN-nCas9-UGI)-gRNA nuclease complex can catalyze site-specific base conversion of zebrafish genome in vivo, we first replaced the Cas9 sequence in the original plasmid with a zebrafish codon-optimized version ${ }^{12}$. To assess the feasibility of its use in zebrafish, four target sites from three genes (twist2, gdf6, and ntl) were selected (Fig. 1a). After injecting BE messenger RNA (mRNA) and related gRNA into one-cell embryos of zebrafish, six embryos at $48 \mathrm{~h}$ post fertilization (hpf) were randomly selected and mixed together for genomic DNA extraction. PCR amplification of the region covering the target site was performed, and the products were used for both sequencing and T-A cloning. Sequencing results of PCR products revealed overlapping signal peaks at the position where the targeted cytidine was located. Outside the targeted cytidine site, no other significant overlapping signal peaks were observed in this region. These results were further confirmed by sequencing individual clones. For twist $2-1,4$ of the 20 colonies carried $\mathrm{C} \rightarrow \mathrm{T}$ conversions, 1 carried a $\mathrm{C} \rightarrow \mathrm{A}$ conversion and the remaining 15 maintained wild-type sequence (Fig. 1b). For gdf6, 4 of the 20 colonies showed $\mathrm{C} \rightarrow \mathrm{A}$ conversions and 1 showed $\mathrm{C} \rightarrow \mathrm{T}$ conversion (Fig. 1c). Finally for $n t l$, C-T conversion was observed in 4 of the 24 colonies (Fig. 1d). These types of base conversions were transmitted through germline and identified in embryos of F1 generation. PCR and sequencing analysis showed that 6 out of 16 F0 injected fish transmitted the expected base change to F1 for twist2-1 and 2 out of 7 for $n t l$.

The p.E75K mutation in twist 2 was previously reported to be the causative mutation of ablepharon macrostomia syndrome

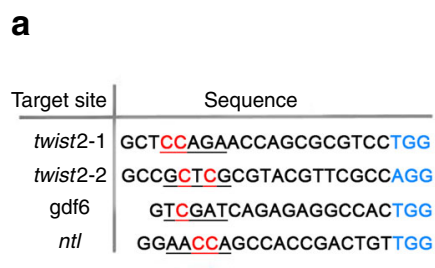

d

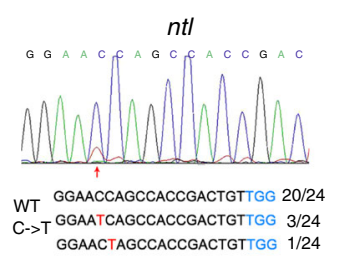

b

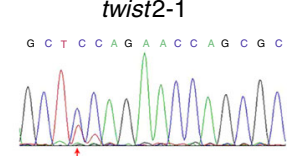

WT GCTCCAGAACCAGCGCGTCCTGG 15/20 C->T GCTTCAGAACCAGCGCGTCCTGG 3/20 GCTTTAGAACCAGCGCGTCCTGG $1 / 20$ C->A GCTACAGAACCAGCGCGTCCTGG $1 / 20$

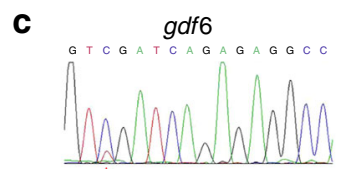

WT GTCGATCAGAGAGGCCACTGG $14 / 20$ C->T GTTGATCAGAGAGGCCACTGG $1 / 20$ C->A GTAGATCAGAGAGGCCACTGG 4/20 INDEL -T ... T-A . ........-CACTGG $1 / 20$

f

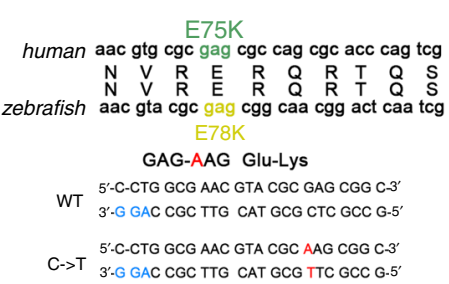

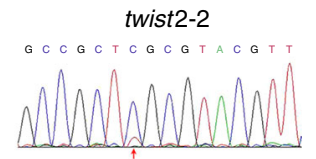

WT GCCGCTCGCGTACGTTCGCCAGG 19/21 C->T GCCGTTTGCGTACGTTCGCCAGG $\quad 1 / 2$ GCCGCTTGCGTACGITCGCCAGG $1 / 21$

Fig. 1 Single-base editing in zebrafish. a The target site sequences. Target sequence (black), PAM region (blue), target sites (red), and target windows (underlined) are indicated. b-d, f Sequencing results at the twist2-1, gdf6, ntl, and twist2-2 targets. e The diagram of mutation of human AMS. Red arrows indicate the overlapped peaks. The substituted bases are marked in red. Red dashes represent the deleted bases in the sequence 
$(\mathrm{AMS})^{13}$. Notably, the conversion of C-T in twist2-2 induced a p.E78K amino-acid change, precisely mimicking the p.E75K mutation found in human (Fig. 1e). Sequencing results showed that 2 out of 21 colonies carried $\mathrm{C} \rightarrow \mathrm{T}$ conversion (Fig. 1f). The p.E78K mutation in twist2-2 was transmitted to the next generation with efficiency of $7.7 \%(2 / 26)$. These results indicate that a zebrafish AMS model precisely mimicking the human mutation can be achieved, suggesting the potential of this system to develop animal models for human disease. Taken together, these data show that base-edited zebrafish can be efficiently generated using this BE-gRNA system.

Base conversion at the tyr locus to mimic OCA mutation. tyr gene has been validated as the most common causal gene for human ocular albinism (OA) and oculocutaneous albinism (OCA). Many point mutations on tyr locus have been verified to cause loss-of-function of the protein, resulting in deficiency in pigment formation. Recently, the mutation p.P301L in tyr has been found in OCA patients (Fig. 2a) ${ }^{14}$. This suggests that the proline at the position 301 may play an important role in the function of the protein. To test this hypothesis, this amino acid needs to be specifically mutated without changing other sequences of this gene. In order to observe the phenotype directly in F0 fish, we co-injected the tyr gRNA/BE mRNA into tyr heterozygous zebrafish embryos and then assessed the base conversion. Because of the codon preference of different species, we could not convert proline to leucine at position 302 in zebrafish. Instead, proline was converted to serine, threonine, or alanine (Fig. 2b, c). The injected embryos showed the phenotype of pigment deficiency in eyes (Fig. 2d). This could reflect the importance of this amino acid to keep tyr function, but it does not exclude the possibility that random indels caused the phenotype. Analysis of germline transmission in F1 embryos identified CCC $\rightarrow$ TCC and CCC $\rightarrow$ TTC (4/25). Screening for other types of base changes is needed to determine which amino-acid change will result in OCA phenotype.

BE system using Cas9-VQR variant. To date, many artificial Cas9 variants with altered PAM specificities have been developed to address the limitation of Cas9 PAM requirment ${ }^{15}$. Among them, Cas9-VQR variant which recognizes 5'-NGA PAM has been shown previously to induce indels in zebrafish and Caenorhabditis elegans ${ }^{16,17}$. We replaced the nCas9 in BE/gRNA system with an nCas9-VQR variant to create a BE-VQR fusion protein. Then three gene loci, twist2, tial1, and urod were tested to see if BE-VQR could induce base conversion at four VQR target a

P301L

human cgt aat cct gga aac cat gac $\begin{array}{llllllllllllll}R & N & P & G & N & H & D\end{array}$ $R \quad N \quad P \quad G \quad D \quad H \quad D$ zebrafish cgc aat $\operatorname{ccc}$ ggg gac cac gac P302L

C

WT GC AAT CCC GGG GAC CAC GAC CGG $15 / 21$ C->T GC AAT TCC GGG GAC CAC GAC CGG 4/21 C->A GC AAT ACC GGG GAC CAC GAC CGG $1 / 21$ C->G GC AAT GCC GGG GAC CAC GAC CGG $1 / 21$ P302A b

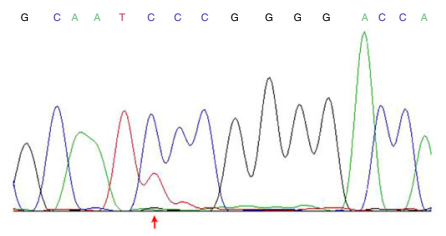

d

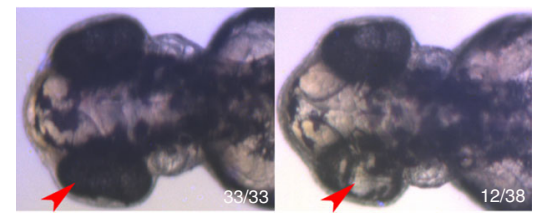

Fig. 2 Induction of amino-acid change at the tyr site in zebrafish. a The diagram of mutation of human OCA. b Sequencing results at the tyr target. c Amino-acid change at the tyr site. d Phenotypes of the injected tyr $+/-$ zebrafish. Red arrows indicate the overlapped peaks. The substituted base and amino acid are marked with red and yellow, respectively. Red arrowhead indicates the deficient eye

a

\begin{tabular}{c|c} 
Target site & Sequence \\
\hline twist2-3 & GCGICCTGGCGAACGTACGCGA \\
urod-1 & GAGATCGTGAGGAGGATGTTGGA \\
urod-2 & GTICCCCTGAAGCTTGCTGTGA \\
tial1 & GGCLTCCGTAACATCCCTGGAGA
\end{tabular}

d

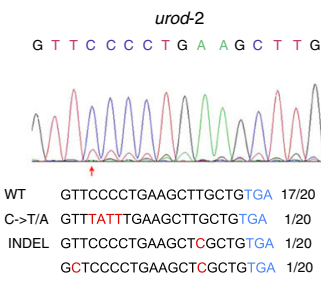

b twist2-3
GC GT C C TG G G G A C G T

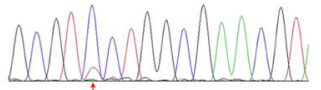

WT GCGTCCTGGCGAACGTACGCGA 16/20 C->T GCGTTTTGGCGAACGTACGCGA 2/20 C $\rightarrow$ T/G GCGTTGTGGCGAACGTACGCGA 1/20 INDEL GCGTGAGAGCGAACGTACGCGA 1/20

e

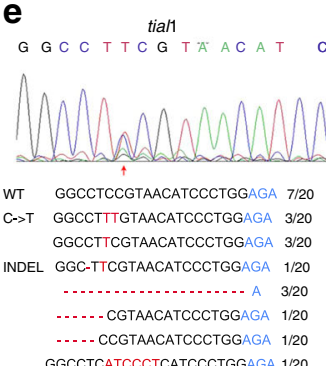

C

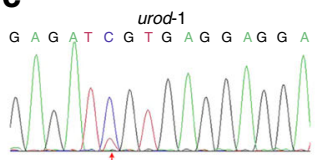

WT GAGATCGTGAGGAGGATGTTGGA 13/20 C->T GAGATTGTGAGGAGGATGTTGGA 6/20 INDEL GAGCATCGTGAGGAGGATGCTGGA 1/20

f G G C C T C $\stackrel{t i a / 1}{\mathrm{C}}$ T A A C A T C C

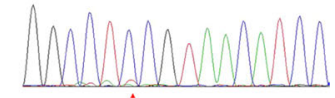

WT GGCCTCCGTAACATCCCTGGAGA 16/20 C $>$ A GGCCTACGTAACATCCCTGGAGA 1/20 C->T GGCCTTCGTAACATCCCTGGAGA 2/20 INDEL GGAAACCGTAACATCCCTGGAGA 1/20

Fig. 3 Base editing by Cas9-VQR variant. a The target site sequences. Target sequence (black), PAM region (blue), target sites (red), and target windows (underlined) are indicated. b-e Sequencing results of the twist2-3, urod-1, urod-2, and tiall targets. $\mathbf{f}$ Sequencing result of tiall site when using dCas9-VQR. Red arrows indicate the overlapped peaks. The substituted bases are marked with red. Red dashes represent the deleted bases in the sequence 
sites (Fig. 3a). PCR sequencing results demonstrated that all targeted loci produced overlapping signal peaks at the base conversion site (Fig. 3b-e). It was noted that urod site2 and tiall had some unexpected lower overlapping signal peaks at other bases apart from the cytidine site (Fig. 3d, e), suggesting indels formation. The BE events at these four VQR sites were confirmed by sequencing colonies (Fig. 3b-e). Collectively, these results demonstrate that BE-VQR/gRNA system is also functional in zebrafish.

Interestingly, the high base conversion rate of tiall site was accompanied with high indel formation: 7 out of 20 colonies carried indels, while 6 out of 20 carried base substitutions (Fig. 3e). Since it was reported previously that replacement of nCas9 in BE by $\mathrm{dCas} 9$ would reduce the indel formation rate ${ }^{8}$, we mutated the nCas9-VQR in BE-VQR to dCas9-VQR and assessed the base conversion rate at the same tiall site. As shown by the sequencing data (Fig. 3f), the base conversion rate dropped from 30 to $15 \%$ compared to the $\mathrm{BE}-\mathrm{VQR}$ injected group. At the same time the indel formation rate also dropped from 35 to $5 \%$. This is consistent with the previous report that using dCas9 decreases indel formation at the expense of lower base conversion efficiency ${ }^{8}$.

\section{Discussion}

To the best of our knowledge, this is the first demonstration that specific base conversion with high efficiency can be achieved in zebrafish without the need of template DNA. This finding further expands the application of the CRISPR-Cas9 system in zebrafish. Consistent with previous results in cultured cells, our results demonstrate that the $\mathrm{BE}$ system can induce cytidine to thymine conversion at specific sites in zebrafish. At the same time, substitution of cytidine to adenine or guanine can also be achieved. The germline frequency of precise modification at the four sites by BE system is $7-37 \%$. This is significantly better than the reported rate of $4 \%$ for $\mathrm{HDR}^{18}$, suggesting this $\mathrm{BE}$ system can be an efficient strategy for single base editing in zebrafish. In the case of BE-VQR system, we detected slightly higher indel rates in zebrafish, but silencing both nuclease domains of Cas9 reduced the indel formation. And recently, this system has also been reported to work in human cells, with efficiency of up to $50 \%{ }^{19}$. The engineered Cas9-VQR variant with 5'-NGA PAM specificities expands the application of this BE system in zebrafish, providing an additional base editor.

For diseases caused by dominant mutations, simple knockout of the gene cannot mimic the function gained by the mutation. In such case, only introduction of the exact same mutation can reproduce the altered protein. Furthermore, many diseases have been shown to be heterogeneous, like OCA. Multiple point mutations of the same gene are found in patients with these diseases ${ }^{14}$. The BE system should be very useful in addressing what mutations are causing factors of the disease, as demonstrated by the tyr p.P301L locus described in our study.

Recently, another BE system using dCas9 fused with PmCDA1 from sea lamprey has been reported to correct point mutations $9,20,21$. Unlike the rAPOBEC1-nCas9-based BE system, the 3-5 bases surrounding the -18 position upstream of PAM sequence are frequently subjected to mutation ${ }^{20}$. We tested this PmCDA1nCas9-based BE system in zebrafish and achieved positive base editing results at lower frequency. Given that zebrafish codonoptimized Cas9 improved the BE system in this study, we speculate that it might be helpful to use a zebrafish cytidine deaminase homologs to replace the rat APOBEC $1^{22}$. However, that is outside the scope of this paper.

Taken together, this BE system is effective in inducing base conversion in zebrafish in a programmable manner. It provides a simple and efficient method for generating zebrafish models that can precisely mimic mutations found in human diseases.

\section{Methods}

Zebrafish husbandry. Wild-type $\mathrm{AB}$ zebrafish and $t y r+/-$ fish were raised and maintained at $28.5^{\circ} \mathrm{C}$ in circulating system. All zebrafish experiments were approved by Institutional Animal Care and Use Committee (IACUC) of Peking University. The reference from IACUC of Peking University is LSCZhangB-1.

BE-gRNA or BE-VQR-gRNA design. The original rAPOBEC1-XTEN-Cas9n UGI-NLS plasmid was obtained from Addgene $(\# 73021)^{8}$. The nCas9 was replaced by a zebrafish codon-optimized version, and a BamH1 recognition site was inserted after the polyA sequence for the purpose of linearization. The D1135V/R1335Q/ T1337R mutation of Cas9 was performed on BE plasmid to obtain the BE-VQR. And the H840A mutation of Cas9 was introduced into BE-VQR to obtain dBEVQR. All the target sites are listed in Supplementary Table 1.

In vitro synthesis of capped mRNA and gRNA. The BE, BE-VQR, and dBE-VQR plasmid was linearized by $\mathrm{BamH1}$, and capped mRNA was synthesized using Ambion mMESSAGE mMACHINE kit. The gRNA template was prepared according to the cloning-independent gRNA generation method ${ }^{23}$. And the primers are listed in Supplementary Table 2. The gRNA was synthesized using Ambion MAXIscript kit (Ambion) and purified using an RNeasy FFPE kit (Qiagen)

Zebrafish microinjection and genotyping. About $2 \mathrm{nl}$ mixture of BE mRNA (300 $\left.\mathrm{ng} \mu^{-1}\right)$ and gRNA $\left(25 \mathrm{ng} \mu^{-1}\right)$ was co-injected into one-cell stage wild-type embryos. Injected embryos were incubated at $28.5^{\circ} \mathrm{C}$ for examination of phenotypes. After $2 \mathrm{dpf}$, embryos that developed normally were collected for genotyping. Genomic DNA was extracted from pools of six randomly collected embryos by alkaline lysis buffer-based DNA extraction. The genomic region surrounding the target site for each gene was PCR amplified and cloned into the pEASY-T1 vector (Transgene). Both the PCR fragments and the colonies were sequenced. Genotyping was performed for all the embryos injected with BE/ tyr gRNA individually. All the primers for detection are listed in Supplementary Table 2. Other injected embryos were raised to F0 adult, and then crossed with wild-type fish. The F1 embryos were collected in three groups, seven embryos each, and genomic DNA was extracted for each group. The fragments covering the target site were amplified and sequenced. F0 founders could be identified from the sequencing result. Each experiment was repeated for three times.

Imaging. Zebrafish embryos were anesthetized with $0.03 \%$ Tricaine (Sigma-Aldrich), and mounted in $4 \%$ methylcellulose. Photographs were taken by a Zeiss Axio Imager Z1 microscope, and processed by Adobe Photoshop CS6software.

Data availability. The authors state that all data necessary for confirming the conclusions presented in the article are represented fully within the article or available from the authors upon request.

Received: 12 January 2017 Accepted: 6 June 2017

Published online: 25 July 2017

\section{References}

1. Cong, L. et al. Multiplex genome engineering using CRISPR/Cas systems. Science 339, 819-823 (2013).

2. Hwang, W. Y. et al. Efficient genome editing in zebrafish using a CRISPR-Cas system. Nat. Biotechnol. 31, 227-229 (2013).

3. Auer, T. O., Duroure, K., De Cian, A., Concordet, J. P. \& Del Bene, F. Highly efficient CRISPR/Cas9-mediated knock-in in zebrafish by homologyindependent DNA repair. Genome Res. 24, 142-153 (2014).

4. Irion, U., Krauss, J. \& Nusslein-Volhard, C. Precise and efficient genome editing in zebrafish using the CRISPR/Cas9 system. Development 141, 4827-4830 (2014).

5. Bedell, V. M. et al. In vivo genome editing using a high-efficiency TALEN system. Nature 491, 114-118 (2012).

6. MacArthur, D. G. et al. Guidelines for investigating causality of sequence variants in human disease. Nature 508, 469-476 (2014).

7. $\mathrm{Zu}, \mathrm{Y}$. et al. TALEN-mediated precise genome modification by homologous recombination in zebrafish. Nat. Methods 10, 329-331 (2013). 
8. Komor, A. C., Kim, Y. B., Packer, M. S., Zuris, J. A. \& Liu, D. R. Programmable editing of a target base in genomic DNA without double-stranded DNA cleavage. Nature 533, 420-424 (2016).

9. Zong, Y. et al. Precise base editing in rice, wheat and maize with a Cas9-cytidine deaminase fusion. Nat. Biotechnol. 35, 438-440 (2017).

10. Shimatani, Z. et al. Targeted base editing in rice and tomato using a CRISPR-Cas9 cytidine deaminase fusion. Nat. Biotechnol. 35, 441-443 (2017).

11. Kim, K. et al. Highly efficient RNA-guided base editing in mouse embryos. Nat. Biotechnol. 35, 435-437 (2017).

12. Jao, L. E., Wente, S. R. \& Chen, W. Efficient multiplex biallelic zebrafish genome editing using a CRISPR nuclease system. Proc. Natl Acad. Sci. USA 110 13904-13909 (2013).

13. Marchegiani, S. et al. Recurrent mutations in the basic domain of TWIST2 cause ablepharon macrostomia and barber-say syndromes. Am. J. Hum. Genet. 97, 99-110 (2015).

14. Khordadpoor-Deilamani, F., Akbari, M. T., Karimipoor, M. \& Javadi, G. Sequence analysis of tyrosinase gene in ocular and oculocutaneous albinism patients: introducing three novel mutations. Mol. Vis. 21, 730-735 (2015).

15. Kleinstiver, B. P. et al. Engineered CRISPR-Cas9 nucleases with altered PAM specificities. Nature 523, 481-485 (2015).

16. Feng, Y. et al. Expanding CRISPR/Cas9 genome editing capacity in Zebrafish using SaCas9. G3 6, 2517-2521 (2016).

17. Bell, R. T., Fu, B. X. \& Fire, A. Z. Cas9 variants expand the target repertoire in Caenorhabditis elegans. Genetics 202, 381-388 (2016).

18. Albadri, S., Del Bene, F. \& Revenu, C. Genome editing using CRISPR/Cas9based knock-in approaches in zebrafish. Methods doi:10.1016/j. ymeth.2017.03.005 (2017).

19. Kim, Y. B. et al. Increasing the genome-targeting scope and precision of base editing with engineered Cas9-cytidine deaminase fusions. Nat. Biotechnol. 35, 371-376 (2017).

20. Nishida, K. et al. Targeted nucleotide editing using hybrid prokaryotic and vertebrate adaptive immune systems. Science 353, aaf8729 (2016).

21. Bibikova, M., Beumer, K., Trautman, J. K. \& Carroll, D. Enhancing gene targeting with designed zinc finger nucleases. Science 300, 764 (2003).

22. Basu, U., Wang, Y. \& Alt, F. W. Evolution of phosphorylation-dependent regulation of activation-induced cytidine deaminase. Mol. Cell 32, 285-291 (2008).

23. Bassett, A. R., Tibbit, C., Ponting, C. P. \& Liu, J. L. Highly efficient targeted mutagenesis of Drosophila with the CRISPR/Cas9 system. Cell Rep. 4, 220-228 (2013).

\section{Acknowledgements}

We thank Zenghou Tang for zebrafish husbandry. This work was supported in part by funding from Science and Technology Program of Shenzhen (JCYJ20150924110425180 and JCYJ20151030170755264) and NIH (R21GM109908). We thank Jenny Lin for editing the manuscript.

\section{Author contributions}

Y.Z., W.Q., So.L., and S.L.: Conceived and designed experiments; Y.Z., W.Q., X.L., J.S., H.H., and H.B.: Performed the cloning, RNA synthesis, injection, and genotyping; W.Q. Performed the imaging. Y.Z., W.Q., and S.L.: Wrote the manuscript.

\section{Additional information}

Supplementary Information accompanies this paper at doi:10.1038/s41467-017-00175-6.

Competing interests: The authors declare no competing financial interests.

Reprints and permission information is available online at http://npg.nature.com/ reprintsandpermissions/

Publisher's note: Springer Nature remains neutral with regard to jurisdictional claims in published maps and institutional affiliations.

(c) (i) Open Access This article is licensed under a Creative Commons BY Attribution 4.0 International License, which permits use, sharing adaptation, distribution and reproduction in any medium or format, as long as you give appropriate credit to the original author(s) and the source, provide a link to the Creative Commons license, and indicate if changes were made. The images or other third party material in this article are included in the article's Creative Commons license, unless indicated otherwise in a credit line to the material. If material is not included in the article's Creative Commons license and your intended use is not permitted by statutory regulation or exceeds the permitted use, you will need to obtain permission directly from the copyright holder. To view a copy of this license, visit http://creativecommons.org/ licenses/by/4.0/.

(C) The Author(s) 2017 